\title{
Coeficiente Intelectual y Edad de Menarquia en Niñas Escolares del Area Norte de Santiago
}

\author{
Dr. Carlos Valenzuelal y Sr. Sergio Trujillo V. ${ }^{2}$ \\ Intellectual Quotient and Age at Menarche
}

\begin{abstract}
The corralation coefficients between the age at menarche (AM) and the manual (MIQ), verbal (VIQ) and total (TIQ) intellectual quotient in a sample of 70 girls from the North Area of Santiago were: $:=0.1004$ (N.S.), $-0.2645(\mathrm{P}<0.05)$ and -0.2020 (N.S.) respectively. These were low correlations if the hypothesis that nutritional factors determine most of the variability of these variables is true; because undemutrition leads to development retardation and intellectual impairment. A girl deviated largely from the regression line. The correlation coefficients calculated excluding this girl were: $r=-0.2417(\mathrm{P}>0.05),-0.4132(\mathrm{P}<0.001)$ and $-0.3485(\mathrm{P}<0.001)$ and $-0.3485(\boldsymbol{P}<0.01)$. It is concluded that researches must be made to find non environmental factor that could explain these weak although significant correlation.
\end{abstract}

Numerosos eventos biológicos de la pubertad están modulados por la interacción de factores genéticos y ambientales, aún poco conocidos ${ }^{1-5}$. Se ha comprobado que la desnutrición además de afectar el desartollo morfológico del individuo; también influiría en diversas funciones fisiológicas y del aprendizaje ${ }^{6-8}$. Así la desnutrición provocaria retardo del desarrollo puberal y una disminución del coeficiente intelectual. Si la mayor parte de la variabilidad poblacional de la edad de menarquia (EM) y del coeficiente intelectual (CI) se debiera a diferencias nutricionales entonces se esperaría que ambas variables se correlacionarían altamente y en forma negativa. Pensando que además de los factores nutricionales, los factores genéticos pudieran modificar EM y $\mathrm{Cl}^{9-10}$, encontramos adecuado estudiar la correlación existente entre ambas variables en una muestra de escolares del Area Norte de Santiago, que participan en un estudio de seguimiento longitudinal.

1 Departamento de Biología Celular y Genética, Facultad de Medicina, División Ciencias Médicas Norte, Universidad de Chile, Casilla 6556, Santiago 7, Santiago - Chile.

2 Alumno de TV Año de Medicina, Facultad de Medl. cina, División Ciencias Médicas Norte, Universidad de Chile.

Trabajo financiado parcialmente por el Fondo Nacional de Ciencias (Proyecto 339) y el Convenio Unidad de Genética - Informática, Hospital José Joaquín Aguirre.

\section{MATERIAL Y METODO}

Se escogió una muestra de niños escolares del Area Norte de Santiago a quienes se realizó un examen semestral de salud, como parte de un estudio de seguimiento longitudinal de crecimiento y desarrollo. A parte de esta muestra se le aplicó una prueba para medir coeficiente intelectual global con la prueba standard de Wescller aplicable al niño (WISC) ${ }^{11}$, obteniéndose resultados para pruebas manuales y vetbales. Esta es una muestra sesgada de la población de niños en seguimiento; niños con problemas escolares fueron pareados con nifios sin problemas de aprendizaje, para constituir una muestra en la que se estudió variables neuropsiquicas ${ }^{8}$.

La edad de menarquia fue obtenida algunos af́os después del estudio neuropsicológico esperando que todos los casos indices hubiesen teni. do su primera menstruación.

\section{RESULTADOS}

La Tabla 1 describe la distribución de la EM; la que presenta forma Gausiana con dos modos aparentes en 4650 y 5050 días. La prueba de $\mathrm{X}^{2}$ para normalidad no fue significativa $(P>0.05)$. El promedio $(\overline{\mathrm{X}})$ de $\mathrm{EM}=4735.2$ dias $(\mathrm{S}=$ 360.8).

La Fig. 1 muestra la distribución de $\mathrm{Cl}$ manual (CIM), CI verbal (CIV) y CI total (CIT). Los tres presentan una forma Gausiana. El promedio (X) de estas variables es: CIM $=86 \%(\mathrm{~S}=13.9)$, CIV $=85.6 \%(\mathrm{~S}=13.9)$ y $\mathrm{CIT}=84.3 \%(\mathrm{~S}=14.4)$. 
Tabla 1.

Distribución de la Edad de Menarquia

Edad de Menarquia

(días)

$$
\begin{aligned}
& 3901-4000 \\
& 4001-4100 \\
& 4101-4200 \\
& 4201-4300 \\
& 4301-4400 \\
& 4401-4500 \\
& 4501-4600 \\
& 4601-4700 \\
& 4701-4800 \\
& 4801-4900 \\
& 4901-5000 \\
& 5001-5100 \\
& 5101-5200 \\
& 5201-5300 \\
& 5301-5400 \\
& 5401-5500
\end{aligned}
$$

La Fig. 2 muestra la correlación entre CIT y EM. Se observa una correlación negativa. Las otras figuras de CIV y CIM con EM respectiva. mente muestran una tendencia semejante al anterior. Se observa un punto cuyas coordenadas son $\mathrm{CI}=46 \%$ y $\mathrm{EM}=3710$ días al que llamaremos $\mathrm{A}$.

En la Tabla 2 se muestran los coeficientes de correlación (r) y las correspondientes curvas de regresión considerando y descartando el Punto A.

$E]$ individuo correspondiente al punto $A$ se aleja mucho de la curya de regresión. La distan. cia de este punto a la curva de regresión calculada excluyéndolo del conjunto de puntos puede ser estudiada por una prueba de "t", que mide el número de errores típicos de estimación que haý entre este punto y el esperando para él, según la curva de regresión ${ }^{12}$. Para CIM se encontró $t=3.72 \quad(P<0.035)$, para CIV $t=4.26$ $(\mathrm{P}<0.01)$ y para CIT $t=4.07(\mathrm{P}<0.01)$. Estas probabilidades estân por encima de las reales ya que los valores de $\mathbf{t}$ cayeron fuera de las Tablas.

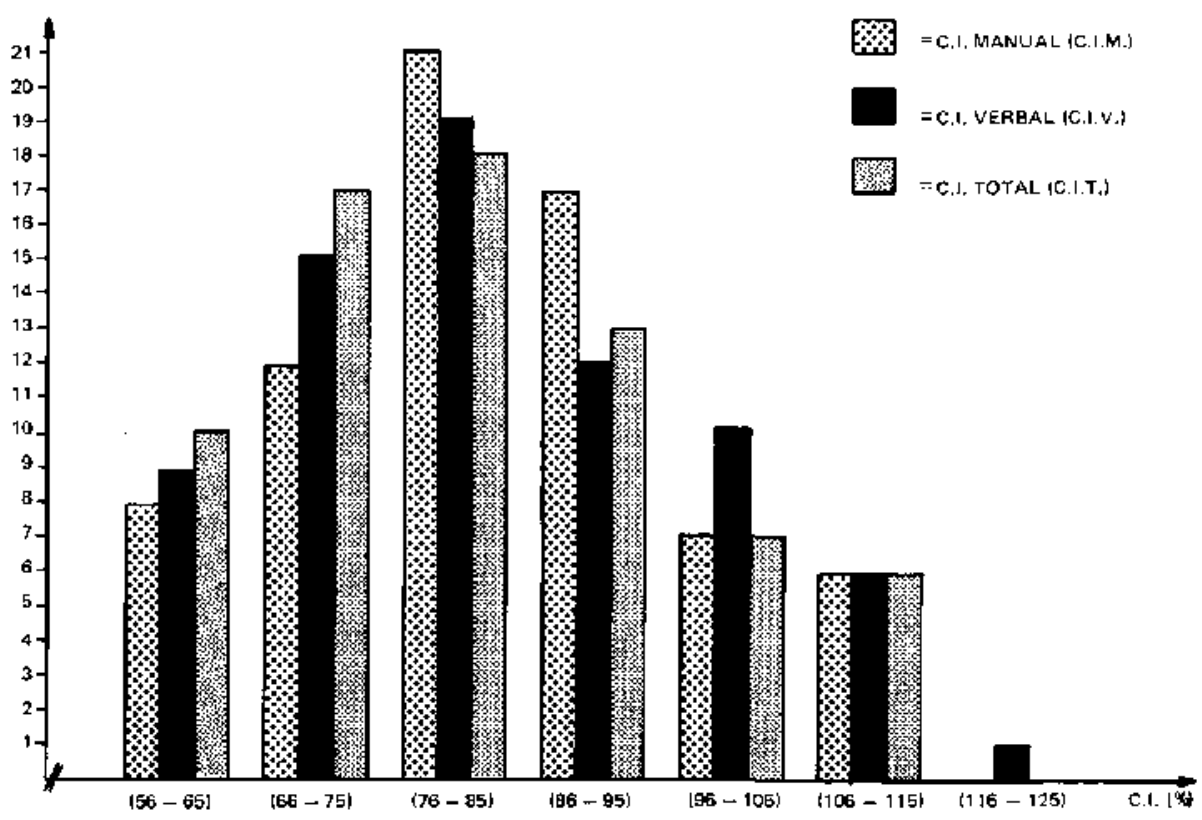

Figura 1.

Distribución đel Coeficiente Intelectual Manual (CIM), Verbal (CIV) y Total (CIT) 


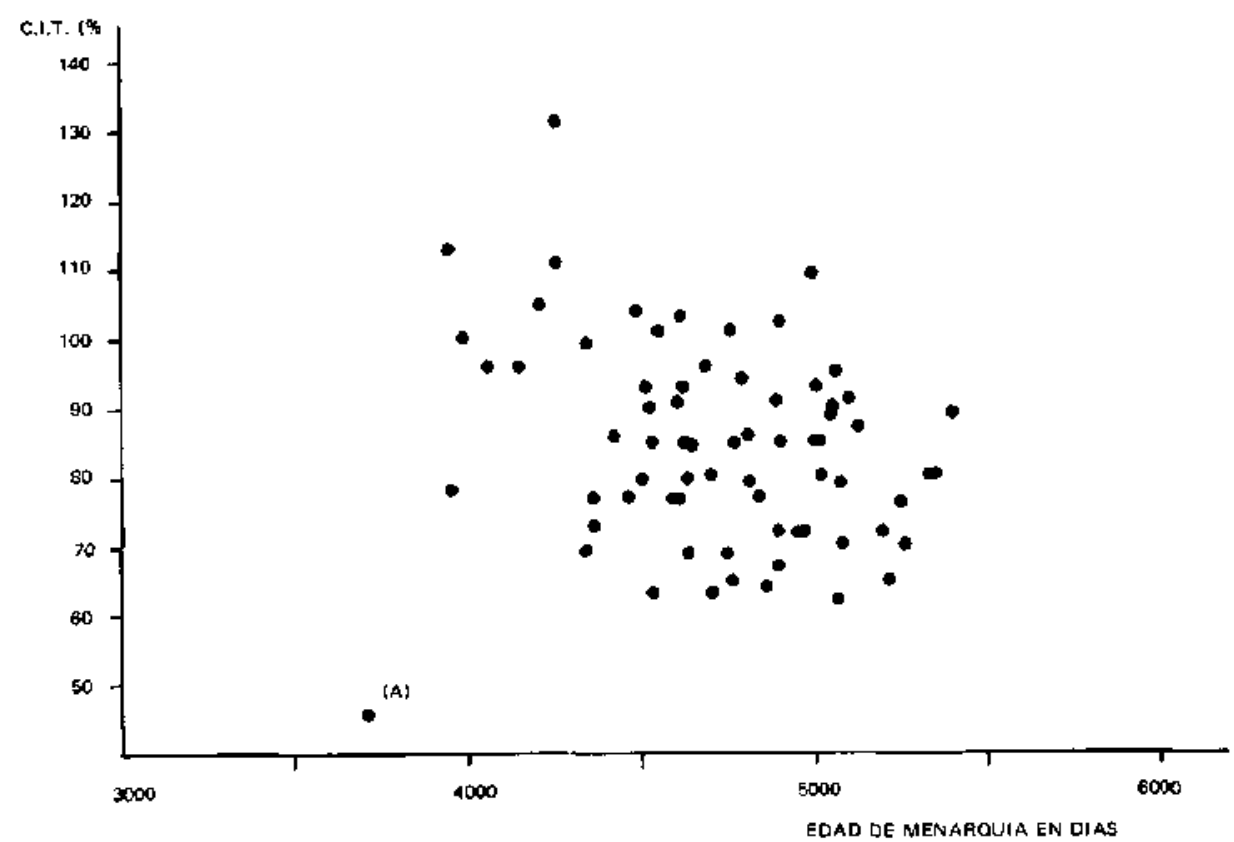

Figura 2.

Correlación negativa entre Coeficiente Intelectual Total (CIT) y Edad en la menatquía (E.M)

Tabla 2.

Edad en la Menarquía y Coeficiente lntelectual

(r) Con punto $\mathbf{A}$

$$
\begin{aligned}
& \mathrm{EM} \text { y CIM }=-0.1004 \text { (N.S.) } \\
& \mathrm{EM} \text { y CIV }=-0.2645 \text { (P }<0.005) \\
& \mathrm{EM} \text { y } \mathrm{CIT}=-0.2020 \text { (N.S.) }
\end{aligned}
$$

(r) Sin punto A

$$
\begin{aligned}
& \text { EM y CIM }=-0.2417(\mathrm{P}<0.05) \\
& \text { EM y CIV }=-0.4132(\mathrm{P}<0.001) \\
& \text { EM y CIT }=-0.3485(\mathrm{P}<0.01)
\end{aligned}
$$

\section{Curvas de regresión}

$$
\begin{aligned}
& Y=104.3387+(-0.0039) \mathrm{X}_{1} \\
& Y=137.4845+(-0.011) \mathrm{X}_{1} \\
& \mathrm{Y}=123.2809+(-0.0082) \mathrm{X}_{1}
\end{aligned}
$$

$$
\begin{aligned}
& Y=131.1992+(-0.0094) X_{1} \\
& Y=168.6579+(-0.0174) X_{1} \\
& Y=153.1143+(-0.0144) X_{1} \\
& Y=C l, X=E M
\end{aligned}
$$

$\mathbf{E M}=$ Edad en la menarquia; $\mathbf{C I M}=$ Coeficiente Intelectual Manual; CIV $=$ Cl Verbal; CIT $=$ CI Total.

\section{DISCUSION}

La representatividad de la muestra y sus seggos están analizados en trabajos previos ${ }^{8}$. Llama la atención la correlación baja entre el valor de $\mathrm{Cl}$ y la EM. Si los factores ambientales fueran los que mayormente influyeran en la modificación de la EM y el CI se esperaría una correlación negativa $y$ alta entre ambas variables; esto es en el caso que deficiencias nutricionales, psicoculturales y socioeconómicas llevaran a un retardo madurativo y a una disminución del CI. En este contexto la correlación baja encontrada indica la participación de factores no ambientales en la variabilidad del CI y de la EM, esto estaría apoyado por lo que a EM se refiere ${ }^{10}$. El individuo $A$ se distancia considerablemente del conjunto de la muestra; su exclusión lleva a valores muy significativos de 
correlación entre los $\mathrm{Cl}$ y $\mathrm{EM}$, aunque los valores de correlación continúan siendo bajos. El caso del individuo A no puede ser explicado por to que se conoce actualmente del efecto de la desnutrición en las dos rariables bajo estudio. Creemos que es importante analizar muestras aleatorias más grandes para confirmar estos ha. llazgos y vislumbrar la naturaleza de los factores no ambientales que podrían estar involucrados en la asociación entre EM y $\mathrm{Cl}$.

\section{RESUMEN}

En una muestra de 70 niñas escolares del Area Norte de Santiago se encontró un coeficiente de correlación entre edad de menarquia (EM) y coeficientes intelectuales (Cl) manual, verbal y total de: $r=-0.1004$ (N.S.), -0.2645 $(P<0.05),-0.2020$ (N.S.) respectivamente, estas correlaciones bajas no corresponden a las esperadas según la hipótesis de que la mayor parte de la variabilidad poblacional del Cl y EM se debería a factores nutricionales, cuya deficiencia llevaría a un retardo puberal y disminución del Cl. Un individuo de esta muestra se distanció significativamente de la curva de regresión, al excluirlo, los coeficientes de correlación se hacen más significativos: $\mathbf{r}=-0.2417 \quad(\mathrm{P}<0.05)$, $-0.4132(P<0.001),-0.3485(P<0.01)$. Se concluye que es necesario investigar factores no ambientales que expliquen esta débil aunque significativa correlación.

\section{REFERENCIAS}

1 Styne, D.M. y' Grumbach, M.M.: Puberty in the male and female: 1ts physiology and disorders. In Repro. ductive Endocrinology. Yen, S.S.C. y Jaffe (Eds.). W.B. Saunders, Philadelphia, pp. 189-240, 1978.

2 Avendaño, A., Valenzula, C.Y., Patri, A. y col.: Antropometría de escolares chilenos del Arca Norte de Santiago. Cuad. Med. Soc. (Santiago, Chile). 16: 5, 1975 .

3 Patri, A.. Sepúlveda, H., Valenzuela, C.Y. y cols.: Estudio longitudinat de crecimiento y desarrolio del nī̄o chileno. Cuad. Med. Soc. (Santìago, Chîle). 20: 11,1979 .

4 Volenzuela, C.Y., Avendeño, A.: Antropometría y maduración sexual de escolares de un Area de Santiago, Chile. Bol. OPS. 87: 113, 1979

5 Marshall, W.A. y Tanner, J.M.: Variations in the pattern of pubertal changes in boys. Arch. Dis. Child. 44: 291, 1970.

${ }^{6}$ ifonckeberg, F.: Jaque al Subdesarrolla. 2a. Edición, Editorial Gabriela Mistral. Santiago, Chile, 1976.

7 Ayell, B.: The impacts of malnutrition on intelligence. Indian Pediatrics. 17 (2): 1980.

8 Manterola, A., Avendaño. A., Valenzuela, C.Y, y cols.: Estudio sobre prevalencia y características de niños con insuficiente rendimiento escolar. Rev. Chil. Ped. 52 (3): 250, 1981.

9 Valenzueta, C.Y.: Diferencias en el crecimiento y desamollo entre niños de Santiago (Chile) y niños europeos y norteamericanos no explicables por diferencias nutricionales. Tesis Doctoral. Santiago, Universidad de Chile, 1977.

10 Rona, R.: Influencia genética-ambiental en la edad de la menarquía en adolescentes de Sartiago. Tesis Doc toral. San tiago, Universidad de Chile, 1972.

11 Wechsler, $D$.: Wechsler in telligence scale for children, The Psycological. Co. New York, 1980.

12 Snedecor, G.W. and Cochran, W.G.: Statistical Methods. 6th Edition. The Iowa State University Press (Ames, lowa, U.S.A.). Pág. 157, 1967. 